\title{
Sequence-dependent effect of gemcitabine and cisplatin on A549 non-small-cell lung cancer cells
}

\author{
YU TANG $^{1}$, YAN WANG ${ }^{1}$ and XIUZHI TENG ${ }^{2}$ \\ ${ }^{1}$ Department of Internal Medicine, Liaoning Tumor Hospital, Shenyang, Liaoning 110042; \\ ${ }^{2}$ Department of Surgery, Center Hospital of Liaoning Electric Power, Shenyang, Liaoning 110015, P.R. China
}

Received January 6, 2013; Accepted May 9, 2013

DOI: $10.3892 / \mathrm{mmr} .2013 .1495$

\begin{abstract}
Cis-diamminedichloroplatinum (cisplatin, CDDP)-containing combination chemotherapy is commonly used for the treatment of non-small-cell lung cancer (NSCLC). 2',2'-Difluorodeoxycytidine (gemcitabine, GEM), an active antineoplastic agent for NSCLC, has been previously reported to be suitable for use in combination with cisplatin in chemotherapy, since their mechanisms may be complementary. In the present study, the sequence-dependent effects of GEM and CDDP were investigated in the NSCLC cell line, A549. Significantly increased rates of inhibition and cell cycle arrest were observed in the group treated with GEM followed by CDDP, and this treatment plan was demonstrated to represent the most efficient treatment protocol for the A549 NSCLC cell line. Results of the present study are consistent with previous studies in other cell lines and are likely to provide important insight for subsequent studies.
\end{abstract}

\section{Introduction}

Non-small-cell lung cancer (NSCLC) is the most common type of lung cancer, with a mortality rate of $>80 \%$. However, the value of chemotherapy in the treatment of NSCLC is controversial. To date, cis-diamminedichloroplatinum (cisplatin, CDDP) remains one of the most important drugs for combination chemotherapy (1). In 1995, a meta-analysis was performed to investigate the impact of CDDP-containing combination chemotherapy $(2,3)$. The results indicated that patients administered with CDDP-containing combination chemotherapy revealed a small but significant $10 \%$ survival benefit at 12 months compared with the best supportive care in advanced NSCLC. As a result of these observations, CDDP-containing combination chemotherapy

Correspondence to: Ms. Yu Tang, Department of Internal Medicine, Liaoning Tumor Hospital, 44 Xiaoheyan Road, Dadong, Shenyang, Liaoning 110042, P.R. China

E-mail: tangyugc@126.com

Key words: cisplatin, gemcitabine, combination chemotherapy, sequence-dependent effect has been commonly used for the treatment of NSCLC; however, the treatment efficacy remains unsatisfactory (4). 2',2'-Difluorodeoxycytidine (gemcitabine, GEM), an active antineoplastic agent for NSCLC, has been previously hypothesized to be suitable for use in combination with CDDP, following observations indicating that these drugs may exhibit complementary mechanisms (5). The sequence-dependent effects of CDDP/GEM treatment remain an important area of clinical and experimental studies, with results demonstrating that various treatment protocols affect the outcome of combination chemotherapy. Previous studies have reported that GEM followed by CDDP represents the most effective treatment in in vitro and in vivo models (6-10). The present study aimed to investigate the sequence-dependent effects of CDDP/GEM treatment on the human non-small-cell lung carcinoma cell line, A549.

\section{Materials and methods}

Chemicals and reagents. GEM and CDDP were purchased from Jiangsu Hansoh Pharmaceutical Co., Ltd. (Lianyungang, China). GEM and CDDP were dissolved in $0.9 \% \mathrm{NaCl}$ and sterile-filtered. Drug concentrations were set according to their respective $\mathrm{IC}_{50}$ values, followed by serial dilutions. In the GEM group, the concentrations used were $0.001,0.01,0.1,1$ and $10 \mathrm{mg} / \mathrm{l}$. In the CDDP group, the concentrations were 0.5 , $1,2,4$ and $8 \mathrm{mg} / \mathrm{l}$, respectively.

Cell culture. A549 cells were grown in monolayers in Dulbecco's modifed Eagle's medium (Gibco, Invitrogen, NY, USA) supplemented with $10 \%$ heat-inactivated fetal bovine serum (Hyclone, Logan, UT, USA), 1\% L-glutamine and 1\% penicillin-streptomycin and incubated at $37^{\circ} \mathrm{C}$ in $5 \% \mathrm{CO}_{2}$. The study was approved by the Institutional Review Board at Liaoning Tumor Hospital (Shenyang, China).

Drug administration. In this study, the cells were divided into six groups, including a control group and five treatment groups. The treatment groups were as follows: GEM, CDDP, GEM followed by CDDP (GEM - CDDP), CDDP followed by GEM (CDDP - GEM) and simultaneous administration of GEM and CDDP (GEM + CDDP). To study the sequence-dependent effects of treatments, cultured cells were exposed to GEM and CDDP alone and in combination. Cells 
were treated with the first drug for $24 \mathrm{~h}$, at concentrations of $\mathrm{IC}_{50}, 75 \% \mathrm{IC}_{50}, 50 \% \mathrm{IC}_{50}$ and $25 \% \mathrm{IC}_{50}$. The cells were then washed with PBS and treated with the second drug, also at concentrations of $\mathrm{IC}_{50}, 75 \% \mathrm{IC}_{50}, 50 \% \mathrm{IC}_{50}$ and $25 \% \mathrm{IC}_{50}$, with the same dilution ratios for both drugs.

Inhibition rate. A549 cells at the exponential growth phase were plated in 96 -well plates $\left(5 \times 10^{3}\right.$ cells in a volume of $100 \mu \mathrm{lmedium} /$ well). Following cell adherence, $100 \mu \mathrm{l}$ GEM (final concentrations, 0.001, 0.01, 0.1, 1 and $10 \mathrm{mg} / \mathrm{l}$ ) and/or CDDP (final concentrations, 0.5, 1, 2, 4 and $8 \mathrm{mg} / \mathrm{l}$ ) were added to the medium. Cells were cultured at $37^{\circ} \mathrm{C}$ for $48 \mathrm{~h}$. Next, medium in the control and drug-containing wells was removed and replaced by $200 \mu \mathrm{l}$ fresh, drug-free MTT medium (0.5 mg/ml; Sigma, St. Louis, MO, USA). Following culture for $4 \mathrm{~h}$ at $37^{\circ} \mathrm{C}$, the medium was replaced by $150 \mu \mathrm{l}$ dimethyl sulfoxide (DMSO; Sigma). Finally, a microplate reader (Tecan, Männedorf, Switzerland) was used to detect the optical density (OD) values in each group at a wavelength of $490 \mathrm{~nm}$. Each group was performed in triplicate in three independent experiments. Inhibition rates and $\mathrm{IC}_{50}$ were calculated based on these values.

Calculation of combination index (CI). CI was calculated using the following formula (11): $\mathrm{CI}=\left(\mathrm{Da} /{ }^{\mathrm{a}} \mathrm{IC}_{50}\right)+\left(\mathrm{Db} /{ }^{\mathrm{b}} \mathrm{IC}_{50}\right)$, where $a$ and $b$ represent two drugs with a similar function. $\mathrm{Da}$ and $\mathrm{Db}$ represent doses that lead to a growth inhibition of $50 \%$. ${ }^{\mathrm{a}} \mathrm{IC}_{50}$ and ${ }^{\mathrm{b}} \mathrm{IC}_{50}$ represent the drug concentration at which an inhibition rate of $50 \%$ was achieved. $\mathrm{CI}<1$ indicated synergism, $\mathrm{CI}>1$ indicated antagonism and $\mathrm{CI}=1$ indicated additivity. The inhibition rate was calculated based on an MTT assay and the $\mathrm{IC}_{50}$ was calculated using logistic analysis.

Cell cycle analysis. Cells were exposed to cisplatin and/or gemcitabine treatment. Following $48 \mathrm{~h}$, cells were washed with ice-cold PBS, recovered by trypsinization, fixed in $70 \%$ cold ethanol and stored at $4^{\circ} \mathrm{C}$. Following centrifugation, $500 \mu \mathrm{l}$ resuspended cells with a concentration of $1.0 \times 10^{6} / \mathrm{ml}$ were collected. Next, propidium iodide (PI) solution was added and incubated for $45 \mathrm{~min}$ at room temperature (in the dark). Flow cytometric analysis was performed with a BD FACScan flow cytometer (Becton Dickinson, San Jose, CA, USA) using a $480 \mathrm{~nm}$ laser as an excitation source and an absorbance wavelength of $630 \mathrm{~nm}$.

Analysis of apoptosis. Apoptotic cells were identified using the BD Pharmingen FITC Annexin V Apoptosis Detection kit (San Diego, CA, USA), according to the manufacturer's instructions. Annexin $\mathrm{V}$ is a $35-36 \mathrm{kDa} \mathrm{Ca}^{2+}$-dependent phospholipid-binding protein that binds phosphatidylserine, a phospholipid continuously exposed in apoptotic cells. Annexin V conjugates with FITC, a fluorochrome, functioning as a sensitive probe for the flow cytometry of cells undergoing apoptosis. Damaged cells were stained by PI. Cells were plated in 6-well plates and divided into 6 groups. Following drug treatment, cells were collected and washed twice with cold PBS. Next, cells were resuspended in binding buffer at a concentration of $1.0 \times 10^{6}$ cells $/ \mathrm{ml}$. In addition, $100 \mu \mathrm{l}$ solution was added to 5 -ml culture tubes together with $5 \mu \mathrm{l}$ FITC Annexin V and $5 \mu \mathrm{l}$ PI. Cells were incubated for $15 \mathrm{~min}$ at room temperature under dark conditions. Binding
Table I. OD values, inhibition rates and CIs of the control and experimental groups.

\begin{tabular}{lccc}
\hline Group & OD value (490) & Inhibition rate & CI \\
\hline Control & $0.986 \pm 0.033$ & - & - \\
GEM & $0.427 \pm 0.034$ & $0.616667 \pm 0.045$ & - \\
CDDP & $0.462 \pm 0.031$ & $0.552941 \pm 0.027$ & - \\
GEM - CDDP & $0.130 \pm 0.013$ & $0.87451 \pm 0.016$ & 0.8007 \\
CDDP - GEM & $0.295 \pm 0.035$ & $0.72451 \pm 0.037$ & 1.1735 \\
GEM + CDDP & $0.254 \pm 0.008$ & $0.74001 \pm 0.017$ & 0.9986
\end{tabular}

GEM, gemcitabine; CDDP, cisplatin; GEM - CDDP, GEM followed by CDDP; CDDP - GEM, CDDP followed by GEM; GEM + CDDP, simultaneous administration of GEM and CDDP; $\mathrm{OD}$, optical density; CI, combination index. Data are presented as the means \pm S.D. of three independent experiments.
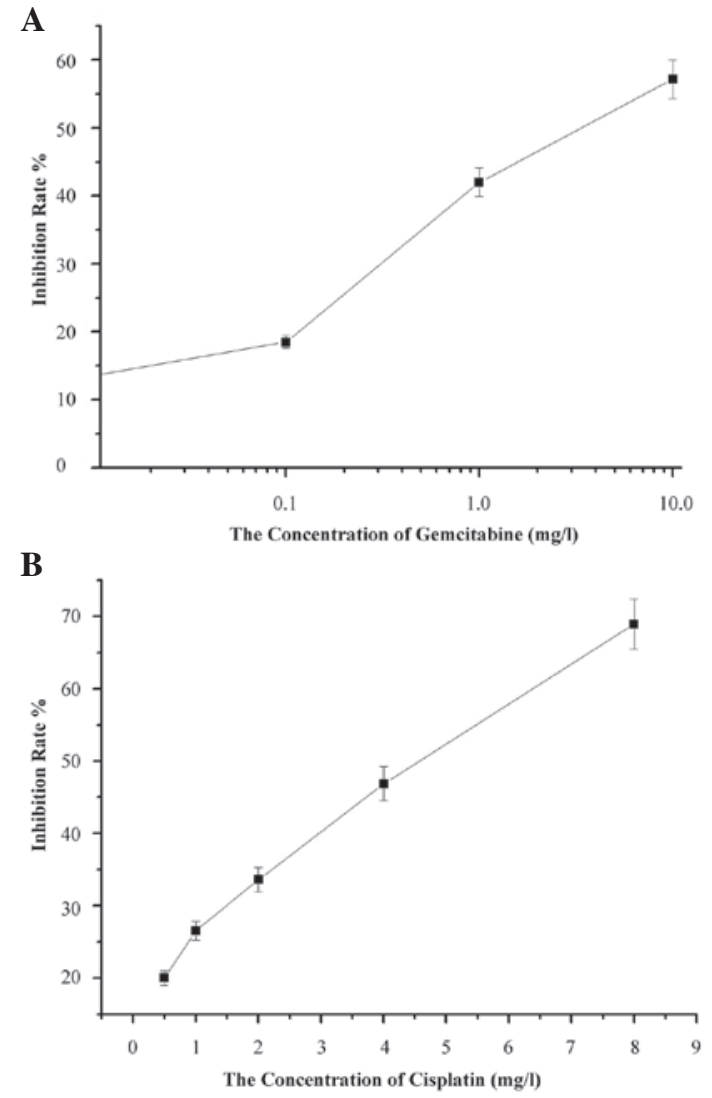

Figure 1. Inhibition rate of A549 cells at various drug concentrations. (A) GEM (0.1-10.0 mg/l) and (B) CDDP (0.5-8.0 mg/l). GEM, gemcitabine; CDDP, cisplatin.

buffer $(400 \mu \mathrm{l})$ was added to the tubes and analyzed using the BD FACScan flow cytometer.

Statistical analysis. The SPSS 13.0 statistical software package (SPSS, Inc., Chicago, IL, USA) was used for statistical analysis. The results of cells treated with different drugs were analyzed by one-way ANOVA. Pearson correlation analysis was used to analyze the dose- and time-dependent effects. $\mathrm{P}<0.05$ was considered statistically significant. 

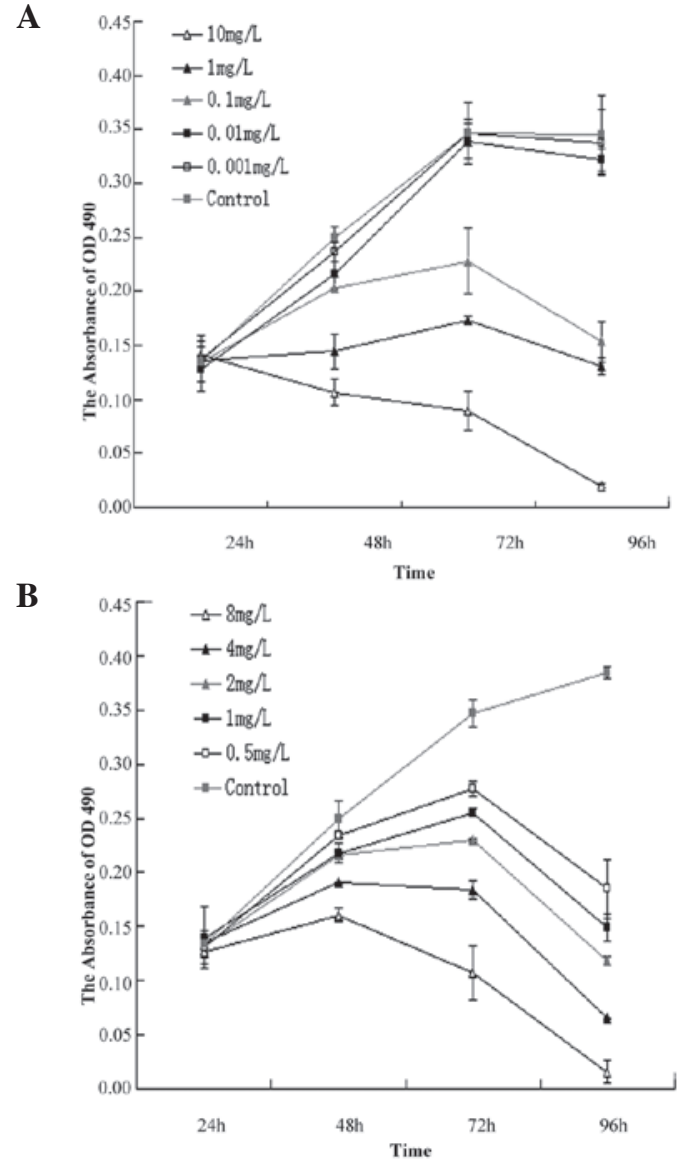

Figure 2. Cell growth curve of A549 cells following drug treatment with (A) GEM (0.1-10.0 mg/l) and (B) CDDP (0.5-8.0 mg/l). OD, optical density; GEM, gemcitabine; CDDP, cisplatin.

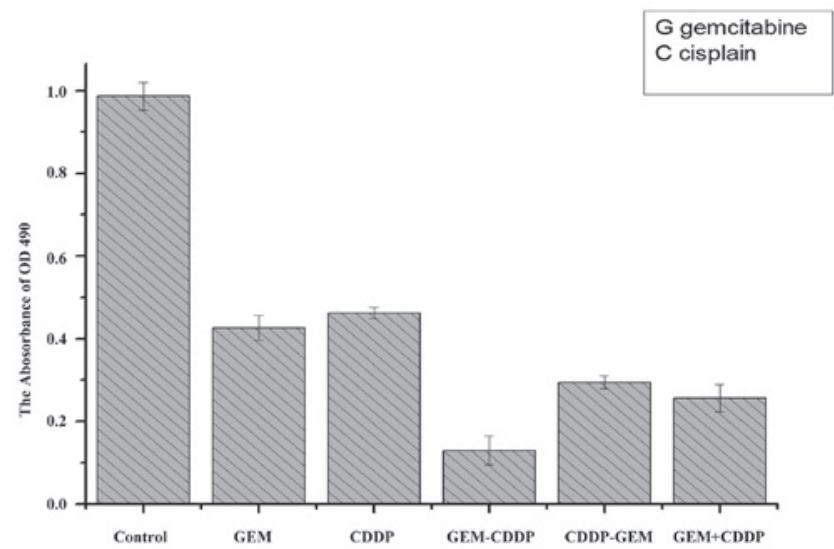

Figure 3. Sequence-dependent effects of GEM and/or CDDP on A549 cells. OD, optical density; GEM, gemcitabine; CDDP, cisplatin; GEM - CDDP, GEM followed by CDDP; CDDP - GEM, CDDP followed by GEM; GEM + CDDP, simultaneous administration of GEM and CDDP.

\section{Results}

Dose and time-dependent effects of GEM and CDDP. To investigate the dose- and time-dependent effects of GEM and CDDP on A549 cells, cells were treated with GEM alone with concentrations of 0.1 to $1 \mathrm{mg} / \mathrm{l}$ or CDDP alone with concen-
Table II. Cell cycle analysis for the control and experimental groups.

\begin{tabular}{llcr}
\hline Group & $\mathrm{G}_{1}(\%)$ & $\mathrm{G}_{2}(\%)$ & \multicolumn{1}{c}{$\mathrm{S}(\%)$} \\
\hline Control & $79.40 \pm 4.2$ & $3.95 \pm 0.61$ & $16.65 \pm 2.37$ \\
GEM & $66.85 \pm 3.23$ & $0.69 \pm 0.16$ & $33.46 \pm 1.98$ \\
CDDP & $62.66 \pm 5.1$ & $1.79 \pm 0.35$ & $35.55 \pm 3.67$ \\
GEM - CDDP & $83.60 \pm 5.2$ & $7.54 \pm 1.04$ & $8.85 \pm 1.07$ \\
CDDP - GEM & $69.40 \pm 3.46$ & $0.00 \pm 0.00$ & $30.60 \pm 2.64$ \\
GEM + CDDP & $69.11 \pm 4.36$ & $0.00 \pm 0.01$ & $30.89 \pm 2.67$ \\
\hline
\end{tabular}

GEM, gemcitabine; CDDP, cisplatin; GEM - CDDP, GEM followed by CDDP; CDDP - GEM, CDDP followed by GEM; GEM + CDDP, simultaneous administration of GEM and CDDP. Data are presented as the means \pm S.D. of three independent experiments.

trations of $0.5,1,2$ and $4 \mathrm{mg} / 1$, respectively. MTT assay was performed to detect cell viability 24,48 and $72 \mathrm{~h}$ after drug exposure. Pearson correlation analysis indicated a dose-effect relationship in the GEM $(\mathrm{r}=0.827 ; \mathrm{P}<0.05)$ and CDDP groups $(\mathrm{r}=0.983 ; \mathrm{P}<0.01)$ within $24 \mathrm{~h}$ (Fig. 1). A time-dependent was also observed in Fig. 2. In the GEM group, $\mathrm{IC}_{50}$ for 24,48 and $72 \mathrm{~h}$ was $3.79 \pm 0.32,1.11 \pm 0.12$ and $0.23 \pm 0.06 \mathrm{mg} / \mathrm{l}$, respectively (Fig. 2A). In the CDDP group, $\mathrm{IC}_{50}$ for 24,48 and $72 \mathrm{~h}$ was $18.58 \pm 2.01,3.78 \pm 0.25$, and $0.76 \pm 0.10 \mathrm{mg} / 1$, respectively (Fig. 2B).

Sequence-dependent effects of GEM and CDDP on the inhibition of cell growth. To investigate the sequence-dependent effects of GEM and CDDP on the inhibition of A549 cells, drug administration with different sequences was performed on cells. When the inhibition rate reached $50 \%$, the concentration for GEM was $0.39 \pm 0.01 \mathrm{mg} / 1$ and $1.32 \pm 0.03 \mathrm{mg} / 1$ for CDDP in the GEM-CDDP group; the concentration for GEM was $0.65 \pm 0.03 \mathrm{mg} / 1$ and $2.21 \pm 0.04 \mathrm{mg} / 1$ for CDDP in the CDDP-GEM group; the concentration for GEM was $0.58 \pm 0.02 \mathrm{mg} / \mathrm{l}$ and $1.80 \pm 0.03 \mathrm{mg} / \mathrm{l}$ for CDDP in the GEM+CDDP group. Inhibition rate and CIs for different groups are presented in Table I. Compared with the control group (Fig. 3), significant inhibitory effects were observed in the GEM and CDDP groups. The sequence-dependent effects of GEM and CDDP were also determined in this study; the GEM - CDDP group revealed the highest rate of inhibition compared with the remaining five groups, indicating that GEM - CDDP chemotherapy exhibited the highest efficiency for killing A549 cells in vivo.

Sequence-dependent effects of GEM and CDDP on apoptosis. The concentration of GEM and CDDP in the combination is based on their respective $\mathrm{IC}_{50}$ values. As demonstrated in Fig. 4, the apoptotic rate in the control group was $2.82 \pm 0.12 \%$. In the GEM group, the apoptotic rate was $14.54 \pm 2.36 \%$, while that of the CDDP group was $15.06 \pm 1.31 \%$. In the combination groups, the rates of apoptosis were $25.72 \pm 3.29,10.99 \pm 1.04$ and $19.24 \pm 3.61 \%$ in the GEM - CDDP, CDDP - GEM and GEM + CDDP groups, respectively. Among these groups, GEM - CDDP revealed 

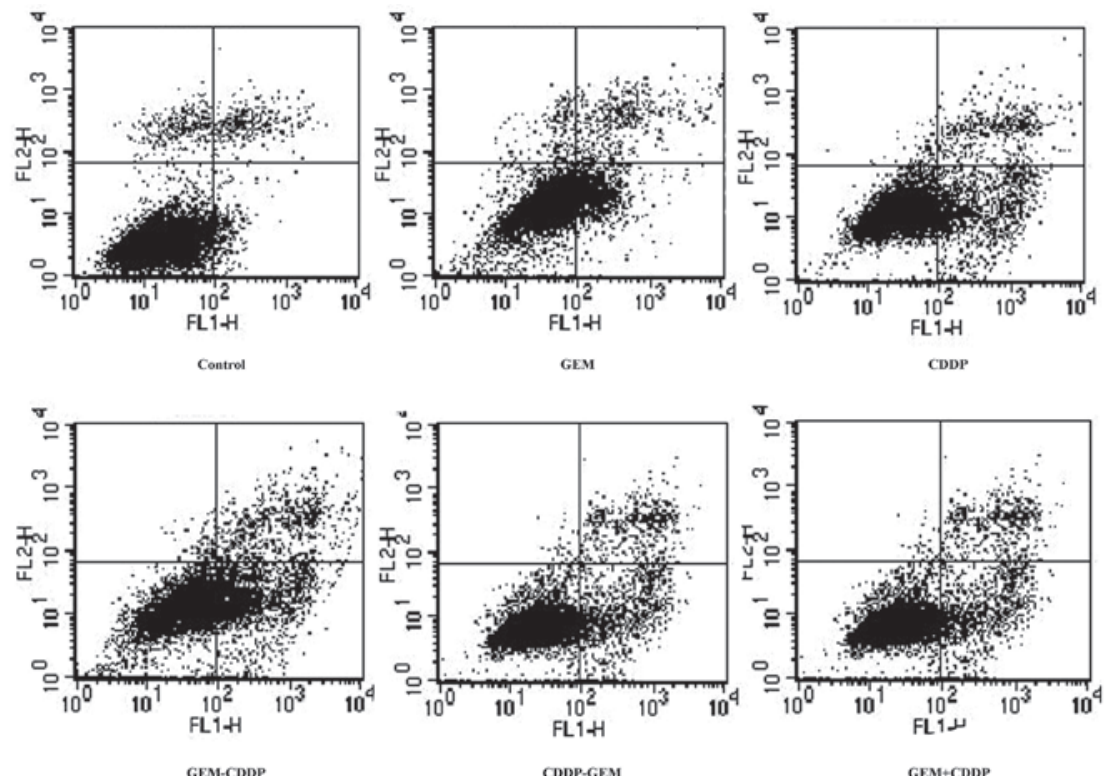

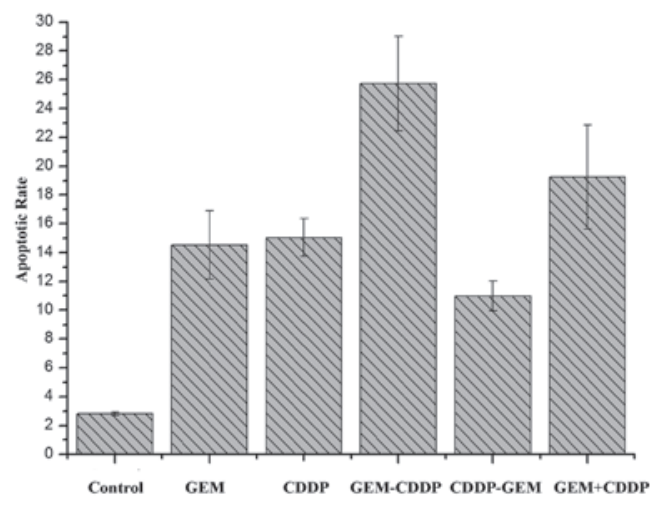

Figure 4. Sequence-dependent effects of GEM and/or CDDP on apoptosis of A549 cells. GEM - CDDP, GEM followed by CDDP; CDDP - GEM, CDDP followed by GEM; GEM + CDDP, simultaneous administration of GEM and CDDP; GEM, gemcitabine; CDDP, cisplatin. Each bar represents the mean \pm S.D. of three independent experiments.

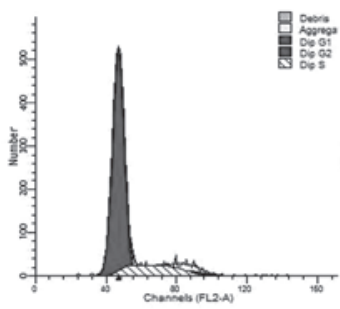

Control

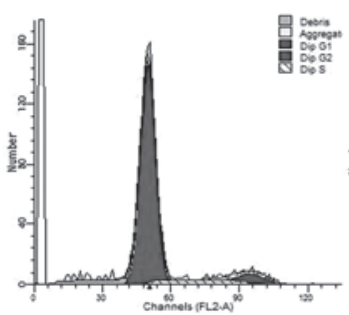

GEM-CDDP

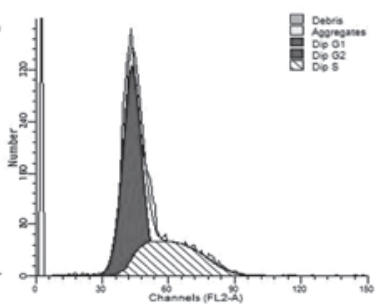

GEM

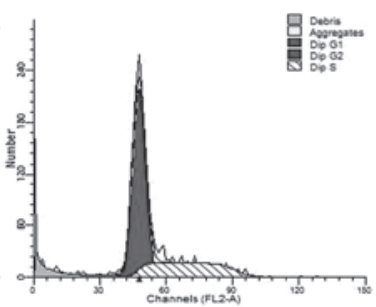

CDDP-GEM

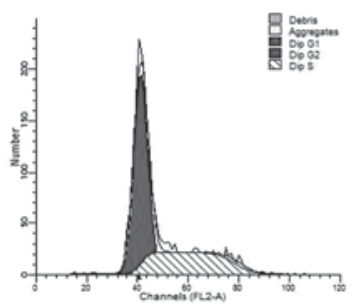

CDDP

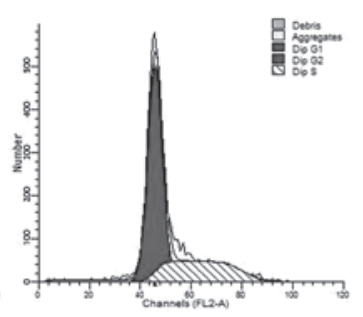

GEM+CDDP

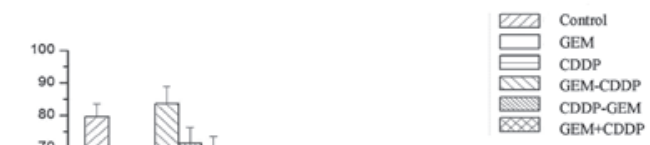

Figure 5. Sequence-dependent effects of GEM and/or CDDP on cell cycle of A549 cells. GEM - CDDP, GEM followed by CDDP; CDDP - GEM, CDDP followed by GEM; GEM + CDDP, simultaneous administration of GEM and CDDP; GEM, gemcitabine; CDDP, cisplatin. Each bar represents the mean \pm S.D. of three independent experiments.

the highest rate of apoptosis compared with the other groups $(\mathrm{P}<0.05)$.

Sequence-dependent effects of GEM and CDDP on the cell cycle. In this study, the sequence-dependent effects of various treatment protocols on the cell cycle were determined (Fig. 5). In groups treated with GEM or CDDP, an increased percentage of cells were arrested in the $S$ phase compared with the control group $(\mathrm{P}<0.05)$. Although the amount of cells arrested in the $\mathrm{S}$ phase in the CDDP group was larger than that in the GEM group, no statistical difference was observed. Consistent results were also detected in the CDDP - GEM, CDDP and GEM groups, where an increased percentage of cells were arrested in the $\mathrm{S}$ phase compared with the control group $(\mathrm{P}<0.05)$. GEM - CDDP was identified to exhibit the highest rate of apoptosis, with the majority of cells arrested in the $G_{1}$ and $\mathrm{G}_{2}$ phases.

\section{Discussion}

CDDP is one of the most widely used anticancer drugs, due to its broad spectrum of activities. CDDP has revealed specific clinical activity for the treatment of NSCLC; however, CDDP induces nephro- and neurotoxicity, ototoxicity, severe nausea, and vomiting (12) and is often withheld from elderly people (13). Therefore, the identification of novel therapeutic 
approaches with mild toxicities is required to improve the clinical outcome of NSCLC. GEM has been hypothesized to be an excellent candidate for combination therapy, as it is associated with moderate side-effects and dose-dependent toxicity. The combination of GEM and CDDP represents an attractive candidate for combination chemotherapy, as the drugs exhibit complementary mechanisms and non-overlapping side effects (14).

Results of the present study indicate that CDDP and GEM exert synergistic effects in vitro, consistent with previous observations (5). In addition, the effect of sequence of drug administration on the outcome of GEM/CDDP treatment was investigated, and it was identified that the administration of GEM followed by CDDP represented the most efficient treatment protocol, consistent with a number of in vitro and in vivo studies (15-18).

GEM functions by blocking nucleic acid synthesis and enzymes involved in the nucleotide biosynthesis pathway. The drug inhibits DNA synthesis and the DNA repair process by reducing the levels of deoxynucleoside triphosphate recruited during DNA synthesis and repair. To date, GEM is considered to be one of the most efficient chemotherapeutic drugs, as it inhibits cell proliferation by preventing cells from progressing from the $G_{1}$ to the $S$ phase. At present, GEM/CDDP combination therapy is preferred for the treatment of advanced NSCLC. Despite previous observations indicating that the administration of GEM/CPPD following an appropriate protocol leads to the synergistic interaction of the drugs (17), high levels of morbidity and a marginal impact on survival time have rendered this combination unsatisfactory. Based on these observations, the aim of the current study was to investigate the sequence-dependent effects of GEM/CDDP treatment in the NSCLC cell line, A549, which is one of the most drug-resistant human tumor cells.

Previous studies have reported sequence-dependent effects of GEM and CDDP in tumor cells. van Moorsel et al (15) reported synergism between GEM and CDDP in NSCLC tumor Lewis lung carcinomas in C57/B16 mice. In addition, Crino et al reported a 54\% efficacy rate in a group treated with GEM followed by CDDP in a phase II NSCLC trial (16). In the trial, 48 previously untreated patients with NSCLC were analyzed. GEM was administered weekly at a dose of $1 \mathrm{~g} / \mathrm{ml}$ and CDDP was administered at a dose of $100 \mathrm{mg} / \mathrm{ml}$ on day 2 of each 28-day cycle. The results indicated that GEM administration followed by CDDP induced a high response rate in stage IIIB and IV NSCLC (16). Finally, antagonism was reported in a study performed with CDDP administration followed by GEM (concentration, $60 \%$ of the $\mathrm{IC}_{50}$ ) for $24 \mathrm{~h}(18)$. Results of the present study are consistent with these observations, providing further evidence for the sequence- and dose-dependent effects of GEM and CDDP therapy on tumor cells.

A number of variables, including the schedule, dose and type of study, are considered to be important for the efficacy of administration of GEM and CDDP combination. However, the mechanisms by which these drugs mediate their effects remain unclear.

In the present study, synergism was observed in the group treated with GEM followed by CDDP at12 h. Antagonism was observed in the group treated with CDDP followed by
GEM at $12 \mathrm{~h}$. Additivity was observed in the group treated with GEM and CDDP simultaneously. Results of previous studies, together with the present study, indicate the presence of sequence-dependent effects of GEM and CDDP treatment, and suggest that these are dependent on dosage, schedule and cell lines to a significant extent. However, at present, no mechanisms accounting for the synergism between GEM and CDDP have been hypothesized. In the current study, analysis of the cell cycle was performed to determine whether the cell cycle is involved in the sequence-dependent effects of GEM and CDDP treatment. Cells treated with GEM or CDDP alone induced cell arrest in the $S$ phase, with increased rates of cell apoptosis. Cells treated with CDDP - GEM or CDDP + GEM exhibited an increased proportion of cells arrested in the $S$ phase, while in the GEM - CDDP group, cells were arrested in the $G_{1}$ and $G_{2}$ phases. The interaction between CDDP and GEM enables CDDP to affect the incorporation of GEM into DNA and RNA in a cell line-dependent manner (19). In addition, a marked effect on DNA synthesis was noted following administration of CDDP. Thus, CDDP may affect the interaction of GEM with DNA. GEM increases the cellular uptake of CDDP and subsequent DNA-platination $(20,21)$. In a previous study, nucleotide excision repair was observed to occur when CDDP was administered and followed by GEM (6). In addition, levels of ribonucleotide reductase, the target of GEM, increased significantly during the inhibition of DNA synthesis. As CDDP is known to inhibit ribonucleotide reductase, the inhibitory effect of CDDP on ribonucleotide reductase may be reduced by GEM metabolism. In studies performed with GEM followed by CDDP, the accumulation of single- and double-strand breaks was significant and intracellular CDDP was hypothesized to generate active products that form DNA intra- and interstrand cross-links $(22,23)$, increasing the probability of the induction of cell apoptosis.

Emerging drugs, particularly molecular targeted therapies, represent novel options for drug combination therapies (24). Specifically, the epidermal growth factor receptor tyrosine kinase inhibitor, gefitinib (25), and histone deacetylation inhibitors $(26,27)$ have been combined with traditional chemotherapy drugs, demonstrating promising efficacies and novel insights into NSCLC therapies.

In conclusion, results of the current study indicate that drug administration sequence is an important variable in the efficacy of drug combination chemotherapy. These observations are likely to provide insight into the synergism of GEM followed by CDDP.

\section{References}

1. Kroep JR, Peters GJ, van Moorsel CJ, et al: Gemcitabine-cisplatin: A schedule finding study. Ann Oncol 10: 1503-1510, 1999.

2. No authors listed: Chemotherapy in non-small cell lung cancer a meta-analysis using updated data on individual patients from 52 randomised clinical trials. Non-Small Cell Lung Cancer Collaborative Group. BMJ 311: 899-909, 1995.

3. No authors listed: Clinical practice guidelines for the treatment of unresectable non-small-cell lung cancer. Adopted on May 16, 1997 by the American Society of Clinical Oncology. J Clin Oncol 15: 2996-3018, 1997.

4. Berghmans T, Paesmans M, Lalami Y, et al: Activity of chemotherapy and immunotherapy on malignant mesothelioma: a systematic review of the literature with meta-analysis. Lung Cancer 38: 111-121, 2002. 
5. Braakhuis BJ, van Dongen GA, Vermorken JB and Snow GB Preclinical in vivo activity of 2',2'-difluorodeoxycytidine (Gemcitabine) against human head and neck cancer. Cancer Res 51: 211-214, 1991

6. van Moorsel CJ, Veerman G, Bergman AM, et al: Combination chemotherapy studies with gemcitabine. Semin Oncol 24 (2 Suppl 7): S7-17-S7-23, 1997.

7. Peters GJ, Ruiz van Haperen VW, Bergman AM, et al: Preclinical combination therapy with gemcitabine and mechanisms of resistance. Semin Oncol 23 (5 Suppl 10): 16-24, 1996.

8. Braakhuis BJ, Ruiz van Haperen VW, Welters MJ and Peters GJ: Schedule-dependent therapeutic efficacy of the combination of gemcitabine and cisplatin in head and neck cancer xenografts. Eur J Cancer 31 A: 2335-2340, 1995.

9. Bergman AM, Ruiz van Haperen VW, Veerman G, et al: Synergistic interaction between cisplatin and gemcitabine in vitro. Clin Cancer Res 2: 521-530, 1996.

10. Tsai CM, Chang KT, Chen JY, et al: Cytotoxic effects of gemcitabine-containing regimens against human non-small cell lung cancer cell lines which express different levels of p185neu. Cancer Res 56: 794-801, 1996

11. Chou TC and Talalay P: Quantitative analysis of dose-effect relationships: the combined effects of multiple drugs or enzyme inhibitors. Adv Enzyme Regul 22: 27-55, 1984

12. McKeage MJ: Comparative adverse effect profiles of platinum drugs. Drug Saf 13: 228-244, 1995.

13. Bunn PA Jr.: Chemotherapy for advanced non-small-cell lung cancer: who, what, when, why? J Clin Oncol 20: 23S-33S, 2002.

14. Voigt W, Bulankin A, Müller T, et al: Schedule-dependent antagonism of gemcitabine and cisplatin in human anaplastic thyroid cancer cell lines. Clin Cancer Res 6: 2087-2093, 2000.

15. van Moorsel CJ, Pinedo HM, Veerman G, et al: Scheduling of gemcitabine and cisplatin in Lewis lung tumour bearing mice. Eur J Cancer 35: 808-814, 1999.

16. Crinò L, Scagliotti G, Marangolo M, et al: Cisplatin-gemcitabine combination in advanced non-small-cell lungcancer: a phase II study. J Clin Oncol 15: 297-303, 1997.
17. Zanellato I, Boidi CD, Lingua G, et al: In vitro anti-mesothelioma activity of cisplatin-gemcitabine combinations: evidence for sequence-dependent effects. Cancer Chemother Pharmacol 67: 265-273, 2010.

18. Voigt W, Bulankin A, Müller T, et al: Schedule-dependent antagonism of gemcitabine and cisplatin in human anaplastic thyroid cancer cell lines. Clin Cancer Res 6: 2087-2093, 2000.

19. Jackson RC: Amphibolic drug combinations: the design of selective antimetabolite protocols based upon the kinetic properties of multienzyme systems. Cancer Res 53: 3998-4003, 1993.

20. Eastman A: The formation, isolation and characterization of DNA adducts produced by anticancer platinum complexes. Pharmacol Ther 34: 155-166, 1987.

21. Bunch RT, and Eastman A: 7-Hydroxystaurosporine (UCN-01) causes redistribution of proliferating cell nuclear antigen and abrogates cisplatin-induced S-phase arrest in Chinese hamster ovary cells. Cell Growth Differ 8: 779-788, 1997.

22. Karagiannis TC and El-Osta A: Double-strand breaks: signaling pathways and repair mechanisms. Cell Mol Life Sci 61: 2137-2147, 2004.

23. Bergstralh DT and Sekelsky J: Interstrand crosslink repair: can XPF-ERCC1 be let off the hook? Trends Genet 24: 70-76, 2008.

24. Pérol M, Chouaid C, Pérol D, et al: Randomized, phase III study of gemcitabine or erlotinib maintenance therapy versus observation, with predefined second-line treatment, after cisplatin-gemcitabine induction chemotherapy in advanced non-small-cell lung cancer. J Clin Oncol 30: 3516-3524, 2012.

25. Hida T, Ogawa S, Park JC, et al: Gefitinib for the treatment of non-small-cell lung cancer. Expert Rev Anticancer Ther 9: 17-35, 2009.

26. Ozaki K, Kishikawa F, Tanaka M, et al: Histone deacetylase inhibitors enhance the chemosensitivity of tumor cells with cross-resistance to a wide range of DNA-damaging drugs. Cancer Sci 99: 376-384, 2008.

27. Donadelli M, Costanzo C, Beghelli S, et al: Synergistic inhibition of pancreatic adenocarcinoma cell growth by trichostatin A and gemcitabine. Biochim Biophys Acta 1773: 1095-1106, 2007. 